\title{
Croissance du taillis de châtaignier en France : premiers résultats
}

\author{
M. BÉDÉNEAU \\ INRA, Station de Sylviculture, Centre de Recherches d'Orléans, Ardon, F 45160 Olivet
}

\section{Summary}

Chestnut coppice growth in France : first results

Total standing woody biomass is studied on sweet chestnut coppice stands distributed throughout France.

In a first step a regression equation was computed to estimate total above-ground biomass of individual trees in relation to their girth at breast height, from 75 trees sampled in Central France. territory.

This equation was then applied to different plots distributed throughout the entire French

A generalized logistic model was then applied to these data, to estimate biomass and height growth in relation to age.

The equations obtained are :

$$
\begin{gathered}
\text { Biomass }\left(\mathrm{t} . \mathrm{ha}^{-1}\right)=124 \times[1-\exp (-0.078 \times \text { age })]^{1.4} \\
\text { Dominant height }(\mathrm{m})=17.9 \times[1-\operatorname{cxp}(-0.049 \times \mathrm{age})]^{10.67}
\end{gathered}
$$

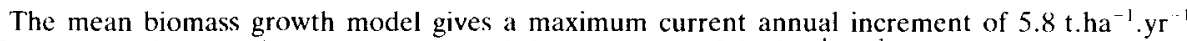
at 5 years, and a maximum mean annual increment of $5.3 \mathrm{t} \cdot \mathrm{ha}^{-1} \cdot \mathrm{yr}^{-1}$ at 9 years. The most productive stands show a current increment which can reach 10 t.ha ${ }^{1} \cdot \mathrm{yr}^{-1}$.

The dominant height of a stand was insufficient to explain variations around the mean biomass growth curve. This is due mainly to differences in growth potential between regions.

Key words: Coppice, biomass, increment, height, simulation, Castanea.

\section{Résumé}

L'évolution de la biomasse ligneuse sèche sur pied est étudiée sur le châtaignier, traité en taillis, sur l'ensemble de la France. Un tarif, permettant de connaître la masse totale du brin en fonction de la circonférence à 1,30 mètre, est d'abord construit à partir de 75 brins prélevés en Région Centre.

Ce tarif est ensuite appliqué à différentes placettes réparties sur l'ensemble du territoire. A partir de ces données on calcule des courbes d'évolution en biomasse et en hauteur, en fonction de l'âge, en leur appliquant un modèle logistique généralisé. 
On obtient respectivement :

$$
\begin{aligned}
& \text { Biomasse (en t.ha' })=124 \times[1-\exp (-0,078 \times \text { âge })]^{1,4} \\
& \text { Hauteur dominante }(\mathrm{en} m)=17,9 \times[1-\exp (-0,049 \times \text { âge })]^{0.67}
\end{aligned}
$$

Pour le modèle moyen d'évolution en biomasse sèche la production courante maximale est de 5,8 t.ha ${ }^{-} \cdot \mathrm{an}^{-1}$ à 5 ans, la production moyenne maximale est de 5,3 t.ha ${ }^{-1} \cdot \mathrm{an}^{-1}$ à 9 ans. Les meilleurs peuplements peuvent atteindre une production courante de l'ordre de 10 tha $^{-1}$.an !

On montre que l'introduction de la hauteur dominante dans un modète de croissance en biomasse ne suffit pas à expliquer les variations entre régions.

Mots clés: Taillis, biomasse, accroissement, hauteur, modèle mathématique, Castanca.

\section{Introduction}

Depuis quelques années les recherches sur les potentialités de croissance des taillis existants sont en nombre de plus en plus élevé (CANnel, 1982; Pardé, 1980). A cela plusieurs explications peuvent être apportées : utilisation dans un but énergétique d'une ressource naturelle renouvelable, mobilisation d'une production délaissée, enfin pour des raisons plus scientifiques: le modèle de croissance des rejets de souche est une particularité intéressante du monde végétal qui pose problème quant à l'explication du phénomène (différenciation des bourgeons, fonctionnement des souches et racines, liaison entre parties aériennes et parties souterraines...).

Cette étude s'inscrit dans les recherches sur la compréhension des lois de croissance de tels taillis : il s'agit de préciser l'évolution de la masse ligneuse tout au long d'une révolution.

Elle concerne le châtaignier (Castanea sp.), espèce présente dans presque toute la France métropolitaine, principalement à l'état de taillis. En effet on estime à 570000 hectares la surface boisée en châtaignier, de cette surface 1300000 mètres cubes sont récoltés annuellement. La ressource sur pied est évaluée à plus de 81 millions de mètres cubes. Le châtaignier est la troisième essence feuillue française en volume sur pied derrière les chênes et le hêtre (Ministère de l'Agriculture, 1985).

Les deux tiers de cette ressource sont en taillis, 95 p. 100 se trouvent en forêt privée. Un réel effort de mobilisation des bois sur pied a actuellement lieu, notamment en essayant d'apporter une haute valeur ajoutée aux produits de la châtaigneraie française (BourGEols, 1987).

Toutefois la biomasse indifférenciée de châtaignier représentera toujours un apport de bois non négligeable, que cet apport soit sous forme de tiges ôtées en éclaircie en vue de balivage ou encore sous forme de rebuts dus à la roulure, l'encre ou toute autre maladie. Or les problèmes de la châtaigneraie varient notamment entre les diverses régions de production, tant en ce qui concerne les débouchés locaux que la façon même de produire (piquets en Normandie, « feuillards » du Limousin...).

C'est pourquoi on a choisi de dresser des tables "d'évolution » en biomasse pour le taillis de châtaignier sur l'ensemble du territoire français. On obtient bien sûr des courbes moyennes qui ne tiennent pas compte de l'effet local.

Il ne s'agit donc que d'une première approche sur les potentialités de production des taillis de châtaigniers existants. 
Comme PAGÈs (1986), on ne fait pas la distinction entre nécromasse et biomasse et on parle plutôt de "masse ligneuse totale sur pied" (il s'agira toujours de masse ligneuse séchée à $105^{\circ} \mathrm{C}$ à l'étuve jusqu'à poids constant).

\section{Matériel et méthodes}

\subsection{Généralités}

Pour la construction de modèles de croissance on a utilisé la méthodologie suivante :

- passage de paramètres simples à mesurer à des biomasses individuelles,

- application de la relation définie ci-dessus à l'ensemble des brins de placettes judicieusement installées,

- extrapolation des données ainsi obtenues dans le temps et l'espace,

- construction proprement dite du modèle de croissance.

Cette démarche amène dans un premier temps à poser certaines hypothèses simplificatrices :

- on considère que les relations permettant d'obtenir la biomasse d'un brin sont invariantes pour l'ensemble des placettes ;

- puisque l'on ignore l'histoire de chaque peuplement on est amené à classer les placettes par ordre chronologique afin d'obtenir pour un âge donné une masse ligneuse sur pied correspondante. Même si le nombre de rotations affecte la productivité, c'est une variable que l'on ne contrôle pas.

\subsection{Tarif "brin"}

Les masses aériennes sèches totales sont estimées selon la méthode décrite par Auclair \& MÉTAyer (1980) sur 75 brins de circonférence à 1,30 mètre comprise entre 3 et 51 centimètres, prélevés en Région Centre.

Une régression non linéaire pondérée (BERGEz et al., 1988) donne l'équation suivante :

$$
\mathrm{b}=0,184+0,00296 \times \mathrm{c}^{2,6}
$$

$\mathrm{b}=$ biomasse sèche du brin $(\mathrm{kg})$,

$\mathrm{c}=$ circonférence du brin $(\mathrm{cm})$.

Le coefficient de corrélation est de 0,989.

Ce coefficient est jugé suffisant pour ne pas introduire de variable supplémentaire, qui pourrait dans la pratique représenter un surcroît de mesures (telle que la hauteur).

\subsection{Tarif "placette»}

On a demandé à divers organismes, par l'intermédiaire du Groupe Châtaignier de l'I.D.F., des données de placettes installées dans des taillis de châtaignier. 
Elles ont respecté le plan d'implantation des placettes permanentes décrit par Decourt (1973), sauf pour la surface minimale qui est de 3 ares : pour chacune d'elle on connait :

- la surface,

- l'inventaire en circonférence de tous les brins, différentes,

- les hauteurs d'au moins 3 des plus gros brins par are, mesurées sur des souches

- l'âge.

Ces placettes sont situées en Normandie, Ile-de-France, Sologne, Limousin, Périgord, Languedoc.

Le tarif décrit précédemment est appliqué à chacun des brins de la placette considérée. La biomasse sur pied est obtenue en faisant la somme des biomasses individuelles.

Les données sont converties en biomasse sèche à l'hectare, exprimée en tonnes.

La hauteur dominante est obtenue en faisant la moyenne des hauteurs des 3 brins les plus gros sur 3 ares.

\subsection{Ajustements}

On a utilisé la technique de régression non linéaire (Tomassone et al., 1983; Bouvier et al., 1985). Les paramètres sont estimés par réitération selon le critère du maximum de vraisemblance. Les calculs ont été effectués à l'aide du logiciel CS-NL développé par le laboratoire de biométrie de Jouy-en-Josas sur système Multics de I'INRA.

\section{Résultats}

\subsection{Modèle de croissance en biomasse}

Pour décrire la croissance en biomasse des peuplements on a utilisé le modèle logistique généralisé, comme PAGÈs (1986) dans les taillis de robinier, qui s'écrit:

$$
\mathrm{Y}=\mathrm{a} \times\left[1-\exp \left[-\mathrm{k} \times\left(\mathrm{t}-\mathrm{t}_{0}\right)\right]\right]^{\mathrm{m}}
$$

dans lequel $\mathrm{Y}$ est la grandeur étudiée et $\mathrm{t}$ l'âge.

Le modèle retenu donne :

$$
\mathrm{B}=124 \times[1-\exp (-0,078 \times \text { âge })]^{1,4}
$$

$\mathrm{B}=$ biomasse sèche (en t.ha ${ }^{\mathrm{T}}$ ).

Les points expérimentaux et la courbe ajustée sont présentés sur la figure 1. La figure 2 montre la courbe d'accroissement courant et la courbe d'accroissement moyen déduites de cet ajustement. 




Ajustement du modèle biomasse (chaque point représente une placette).

Ajusted curve and experimental biomass data (each point represents one plot).

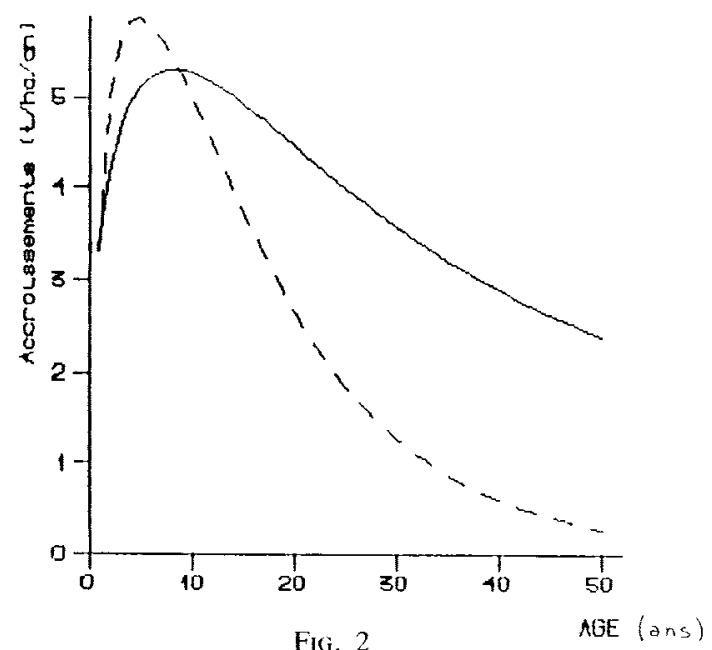

Accroissement courant (ligne hachurée), accroissement moyen (ligne pleine) en biomasse calculés à partir de la courbe moyenne.

Current (dashed line) and mean (solid line) biomass annual increment, computed after the mean curve.

\subsection{Modèle de croissance en hauteur}

Le même type de modèle a été employé pour décrire la croissance en hauteur. On obtient :

$$
\mathrm{H}_{0}=17,9 \times[1-\exp (-0,049 \times \text { âge })]^{0,67}
$$

$\mathrm{H}_{0}=$ hauteur dominante (en $\mathrm{m}$ ). 
Les points expérimentaux et la courbe ajustée sont présentés en figure 3. A partir de ce modèle on calcule l'accroissement courant en hauteur, la courbe est présentée sur la figure 4.

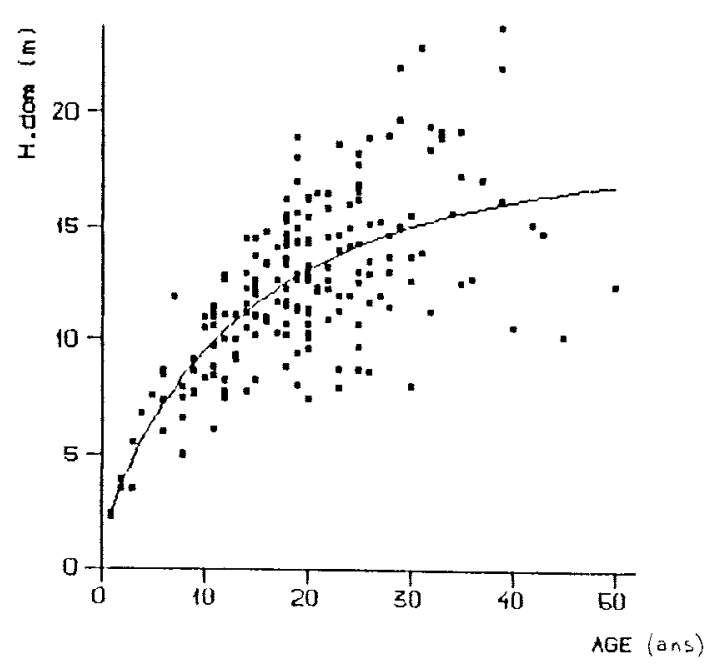

FIG. 3

Ajustement de la courbe de croissance en hauteur (chaque point représente une placette). Ajusted curve and experimental data for dominant height (each point represents one plot).



Fig. 4

Accroissement courant en hauteur du taillis de châtaignier (déduit de la courbe moyenne du modèle de croissance en hauteur).

Current dominant height increment for chestnut coppice, computed after the mean curve. 


\subsection{Modèle de croissance en biomasse intégrant la hauteur}

Il est tentant de rapprocher les deux modèles précédents, en intégrant la hauteur dans un troisième modèle. En effet, si l'on peut considérer la hauteur comme un indicateur de la "fertilité " d'une station, la relation alors obtenue doit permettre un classement des peuplements de châtaigniers.

On a procédé de la même façon que PAGÈs (1986) en prenant le rapport hauteur calculée/hauteur observée $(\hat{\mathrm{H}} / \mathrm{H})$ et en le confrontant aux résidus du modèle moyen en biomasse, décrit ci-dessus (fig. 5).

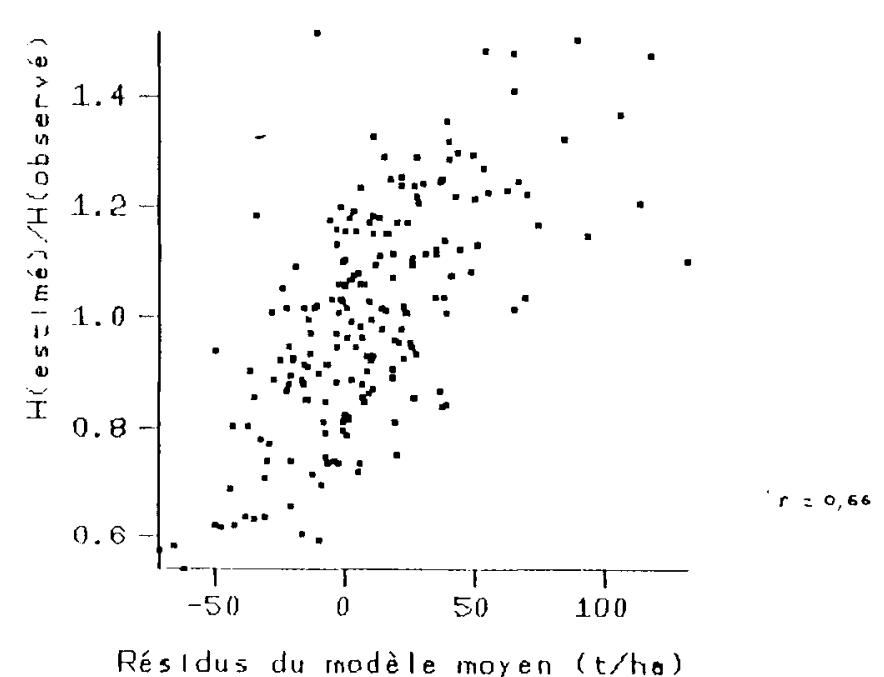

FIG. 5

Relation entre résidus du modèle biomasse et rapport: $H$ estimée/H observée.

Relation between residuals of the biomass model and estimated/observed height ratio.

On constate que les résidus du modèle "biomasse " sont bien corrélés avec le rapport $\hat{\mathrm{H}} / \mathrm{H}(\mathrm{r}=0,66)$. Si on introduit ce rapport dans un modèle à quatre paramètres, on obtient :

$$
\mathrm{B}=(\hat{\mathrm{H}} / \mathrm{H})^{0,161} \times 126[1-\exp (-0,077 \times \text { âge })]^{1,4}
$$

$\mathrm{B}=$ biomasse sèche $\left(\mathrm{t} \cdot \mathrm{ha}^{-1}\right)$.

Le test statistique du rapport de vraisemblance amène à rejeter ce modèle, c'est-àdire que dans ce cas précis la hauteur dominante ne suffit pas à expliquer les variations de la population.

La figure 6 montre les variations de hauteur dominante selon les régions. On remarque que s'il existe une bonne liaison entre la hauteur et l'âge, la nature de cette relation diffère selon les régions: les placettes de Sologne ne croissent guère au-dessus 


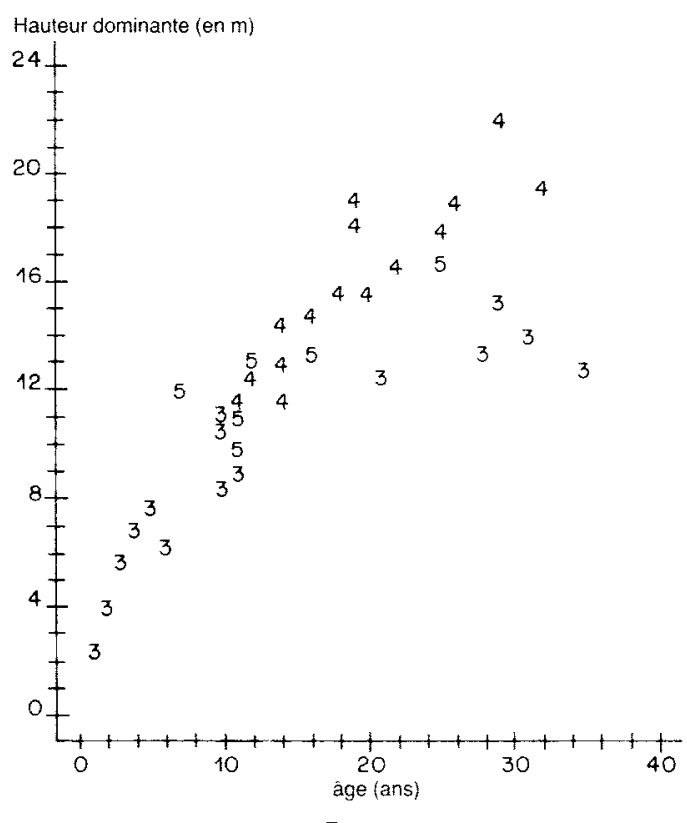

Fici, 6

Relations Hauteur - âge pour différentes régions: 3: Sologne, 4: Périgord, 5: Normandie.

Retation between dominan height and age for various regions: $3:$ Sologne, 4 : Périgord, 5: Normandy.

de 15 mètres, en revanche les placettes de Normandie ou du Périgord semblent encore se développer au-delà de 22 mètres.

Ceci suggère qu'il existe une réelle différence entre régions.

\section{Discussion-conclusion}

Il est évident que nos modèles expliquent insuffisamment la croissance du taillis de châtaignier à l'échelle à laquelle elle a été traitée. On peut trouver à cela quelques explications :

- Modèles : l'échantillon de brins ne recouvre peut être pas toute la variabilité de biomasse, c'est-à-dire que l'hypothèse d'invariance de la relation biomasse-circonférence du brin n'est pas nécessairement valide. Il existe sans doute une grande variation de la densité du bois, ce qui nécessiterait la construction d'un tarif par région ou même par placette. On a cependant jugé intéressant dans un premier temps de présenter ces résultats.

On n'a pris en compte ici, pour expliquer la croissance du taillis de châtaignier, que la variable âge. L'extrême diversité des placettes nécessiterait l'introduction des 
paramètres plus pertinents, mais moins aisés à mesurer puis à modéliser, tels que la densité de brins ou de souches, ou des facteurs de forme et d'espacement.

- Echantillonnages : l'échantillon de placettes n'est vraisemblablement pas représentatif des régions considérées, en ce sens que certaines régions présentent des placettes très productives, d'autres des placettes moins productives, alors que ces deux types de placettes peuvent être présents simultanément. Ces modèles paraissent cependant satisfaisants : les asymptotes sont suffisamment élevées - tant sur le modèle biomasse que sur le modèle hauteur - pour admettre une croissance mesurable, même après 50 ans, ce qui avait été montré par Chevrou (1986).

Un résultat inattendu de cette étude est la courbe de croissance en hauteur : contrairement à celle de la futaie, la croissance en hauteur est maximale dès la première année et ne fait que décroître par la suite. Ceci sous-entend un mode de fonctionnement différent de celui de la futaie, pouvant être expliqué par des phénomènes morphogénétiques, soit au niveau de la partie aérienne (évolution des bourgeons aériens à l'intérieur de la souche), soit au niveau de la partie souterraine (réserves glucidiques dans la souche et les racines).

Cette étude fournit des courbes d'évolution de biomasse ligneuse sur pied: on obtient pour le peuplement moyen une production moyenne annuelle de 5,3 tonnes de matière ligneuse sèche à l'hectare à l'âge de 9 ans. Les peuplements les plus productifs de l'échantillon peuvent atteindre une production courante de 10 t.ha ${ }^{-1}$. an " .

Rollinson \& Evans (1987) publient des chiffres de production pour le châtaignier dans le Sud de l'Angleterre de 400 tonnes de matière ligneuse «fraîche » à 30 ans, ce qui équivaut après conversion à environ $6,6 \mathrm{t} \mathrm{ha}^{-1} \cdot \mathrm{an}^{-1}$ de biomasse sèche. Il est à noter que ces chiffres ne se rapportent qu'aux taillis anglais les plus productifs, qui couvrent environ 20000 ha. Les taillis médiocres, n'étant pas entretenus n'entrent pas dans les tables (Auclair, comm. pers.).

Nos résultats montrent que le châtaignier se place parmi les espèces les plus productives, si l'on considère les traitements sylvicoles traditionnels (taillis, t.s.f., futaie). D'autres types de sylviculture, par exemple les taillis à courte rotation, peuvent avoir des productions plus élevées, mais en culture très intensive (AuclaIr, 1986).

Ces chiffres montrent la cohérence de nos résultats et la nécessité de poursuivre de telles études, en affinant les échantillonnages, et en mesurant des variables plus pertinentes.

Reçu le 4 décembre 1987.

Accepté le 10 février 1988.

\section{Remerciements}

Ce travail a nécessité la collaboration de plusieurs organismes et je remercie plus particulièrement : $M^{\text {Hc }}$ Bourgeois (I.D.F.), MM. Cabannes (C.R.P.F. Languedoc-Roussillon), Duyck et Bercovici (C.R.P.F. Normandie), Lefièvre (C.R.P.F. Aquitaine), Pfendt (C.R.P.F. Midi-Pyrénées), Guittet (Université d'Orsay), Bouchon, Ravart, Ranger, Nys (INRA Nancy), Cabanettes (INRA Orléans). 


\section{Références bibliographiques}

Auclatr D., 1986. Productivité des espèces ligneuses forestières. Biomasse Actualités, 9, 26-34.

Auclatr D., Métayer S., 1980. Méthodologic de l'évaluation de la biomasse aérienne sur pied et de la production en biomasse des taillis. Acta accol., Ecol. appl., 1 (4), 357-377.

Bergez J.E., Bisch J.L., Cabanettes A., Pagès L, 1988. Un programme simple de régression non linéaire pondérée adapté aux estimations de biomasse forestière. Ann. Sci. For. (soumis pour publication).

Bourgeors C., 1987. Le châtaignier, des taillis à ne pas négliger. Forêt-Entreprise, 44 ; Dossier détachable, $19 \mathrm{p}$.

Bouvier A., Gelis F., Huft S., Messean A., Neveu P., 1985. Manuel d'utilisation de CS-NL. INRA, Laboratoire de Biométric, Jouy-en-Josas, $180 \mathrm{p}$.

Cannei. M.G.R., 1982. World forest biomass and primary production data. London, Academic Press, $391 \mathrm{p}$.

Chevrou R.B., 1986. Modélisation de l'évolution des peuplements d'après les données de l'Inventaire Forestier National. I.F.N., Montpellier, 18 p.

Décourt N., 1973. Protocole d'installation et de mesures des placettes de production semipermanentes. INRA-C.N.R.F., Nancy, 25 p.

Pagìs L., 1986. Lois de croissance en biomasse du taillis : le robinier dans le Val-de-Loire. Ann. Sci. For., 43 (4), 533-550.

Pardé J., 1980. Forest biomass. For. Abstr., 41 (8), 343-362.

Roll.inson T.J.D., Evans J., 1987. The yield of sweet chestnut coppice. Forestry Commission Bulletin, (64), 20 p., Her Majesty's Stationery Office, Londres.

Tomassone. R., LFsquoy E., Miller C., 1983. La régression, nouveaux regards sur une ancienne méthode statistique, Masson, $180 \mathrm{p}$. 